\title{
Electric-Thermo-Mechanical Analysis of Joule Heating in Dilatometric Specimens
}

\author{
Martin Herrejón-Escutia ${ }^{1}$ - Gildardo Solorio-Díaz ${ }^{1}$ - Héctor Javier Vergara-Hernández ${ }^{2}$ - \\ Edgar López-Martínez ${ }^{3}$ - Gerardo Marx Chávez-Campos² - Octavio Vázquez-Gómez2,4,* \\ ${ }^{1}$ Michoacan University of Saint Nicolas of Hidalgo, Mechanical Engineering, Mexico \\ ${ }^{2}$ Morelia Institute of Technology, Mexico \\ 3 University of the Isthmus, Campus Tehuantepec, Mexico \\ ${ }^{4}$ National Council of Science and Technology, Mexico
}

A mathematical model of Joule heating was developed for an AISI 304 stainless steel in a hollow cylinder dilatometric specimen. The model was developed by means of creating a balance of energy by coupling the generation term due to the Joule heating and the thermal expansion of the specimen. A Newtonian heating system was assumed for a volume element, and it was resolved by means of the finite differences method, generating its own computer code in the Scilab free-license software. The model considers the thermophysical and electrical properties of steel, depending on the temperature. Thermal interactions at the boundary and the linear thermal expansion coefficient were determined by solving the inverse heat conduction problem (IHCP) using the thermal profile and thermal expansion measured experimentally by means of a direct heating device. The model was validated by comparing the thermal response and experimental thermal expansion with simulated responses for different heating rates.

Keywords: mathematical model, continuous heating, Joule effect, dilatometry, AISI 304 stainless steel

Highlights

- A mathematical model of Joule heating was developed to predict the thermal history and expansion of a dilatometric specimen.

- Thermal boundary conditions were determined for the Joule heating of a dilatometric specimen by solving the Inverse heat conduction problem.

- $\quad$ Coefficient of linear thermal expansion dependent of the temperature was determined by solving the mathematical model and develop of an experimental device of Joule heating.

- $\quad$ The mathematical model was satisfactorily validated comparing the thermal history and strain percentage by dilatation with the experimental measurements as a function of the heating rate.

\section{INTRODUCTION}

Dilatometry is one of the most commonly used thermal analysis techniques in the study of solid-solid phase transformations in ferrous alloys [1] and [2], as it makes it possible to monitor (in real time) the microstructural evolution of a phase in terms of the changes in volume that occur during a thermal cycle. When a material undergoes a phase transformation, the crystal structure is modified, and this is manifested as a positive (expansion) or negative (contraction) volume change. This analysis technique makes it possible to determine the critical transformation temperatures that can be used for constructing isothermal transformation (IT) diagrams and/or continuous cooling and continuous heating transformation (CCT and CHT) [1] to [4]. These diagrams are of practical interest in various metallurgical applications, such as the simulation and design of heat treatments, in the prediction of mechanical properties, and in the formulation of mathematical models to resolve the thermal and microstructural fields during the formation or decomposition of a phase [5] and [6]. Many of these studies use commercial dilatometry equipment to determine transformation kinetics through dilatometric specimens of different shapes and sizes, for example, solid cylindrical shape, hollow, or flat in small sizes, ensuring that the changes in volume obtained are due solely to the phase transformation or the internal changes in the material and not to distortion caused by the thermal shock in the heating and quenching stages. Likewise, the dilatometry devices can use different heating media such as magnetic induction, thermal radiation, and direct current or Joule heating [3], [7] and [8]. This latter medium is characterized by providing thermal homogeneity in the material and good control of the heating rate (HR), which can remain constant for the whole heating stage. Another characteristic of Joule heating is that heat generation in the material is internal, which provides a quasi-linear relationship between the current and the temperature, provided no phase transformation exists [9], unlike 
radiation or induction heating in which the behaviour of the temperature depends on the electrical resistance and the refractory material of the furnace (radiation), or the frequency and magnitude of the electric current, as well as the type of inductor (magnetic induction). Another advantage of the direct methods is that they make it possible to obtain an alternate measurement on the most accurate and exact temperature at which the material acts as an electric heating resistance, which can be monitored in real time depending on voltage and intensity. However, because the material is the electrical resistance of the circuit, it is difficult to control the system efficiently. For that, a prior thermoelectric characterization is needed before obtaining the optimal control parameters, because the materials have different thermal and electric properties. In this paper, a mathematical model of Joule heating was implemented with hollow cylindrical dilatometric specimens to predict history and thermal expansion. The purpose of the model is to understand and estimate the thermoelectric behaviour of conductor materials in a direct experimental heating device.

\section{MATHEMATICAL MODEL}

\subsection{Problem Description}

Fig. 1 shows the dimensions of a hollow cylindrical dilatometric specimen of AISI 304 stainless steel, heated using the Joule heating process. The selection of material mainly owes to the fact that AISI 304 steel does not have internal changes to its structure, such as phase transformation, in the operating temperature range $\left(250{ }^{\circ} \mathrm{C}\right.$ to $\left.850{ }^{\circ} \mathrm{C}\right)$. The specimen has a $1-\mathrm{mm}$ thick wall and is subjected to heating by the Joule effect under natural convection conditions on the outer surface of the specimen. Under these conditions, the Biot number is $4.5 \times 10^{-4}$; consequently, a Newtonian heating system in the radial direction is considered for the problem. Taking into consideration that it is a Newtonian system, it is expected that the temperature gradient in the radial direction should be negligible, creating a totally isothermal zone in this direction. During heating, the specimen expands due to the thermal expansion of the material, which is associated with the thermal strain generated. Meanwhile, the specimen is attached to a system of jaws that are used as power supply terminals and cooled though a moderate internal water flow, which makes the temperature profiles parabolic and symmetric in the axial direction, and ensures that the temperature reaches its highest values in the middle part of the specimen. This temperature is representative of the amount of energy generated in the system by the Joule heating.

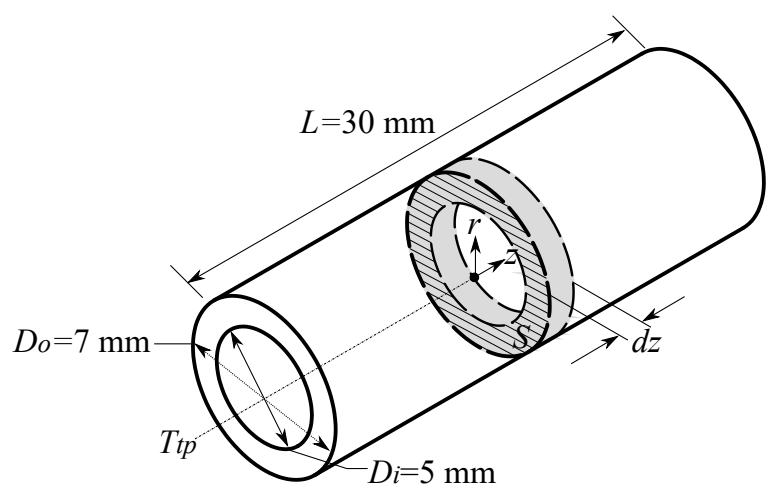

Fig. 1. Hollow cylindrical specimen heated by the Joule effect

\subsection{Thermoelectric Model}

An energy balance inside the isothermal zone indicated by the volume elements $d z$ was made based on Fig. 1 , considering the heat generation and accumulation terms as well as heat loss due to convection through the specimen walls:

$$
Q_{a}=Q_{g}-Q_{l},
$$

where the terms $Q_{a}, Q_{g}$ and $Q_{l}$ are accumulated heat, the generated heat and the lost heat by convection, respectively. The accumulated heat is defined based on the change of temperature with regard to the time and thermophysical properties of the material such as:

$$
Q_{a}=\rho C_{p} V \frac{d T}{d t},
$$

where $T$ and $t$ are heating temperature and time, respectively, $\rho$ is density, $C_{p}$ is specific heat and $V$ is the element volume. In the case of the hollow cylinder, the volume is defined through the transverse area $S$ which is a function of the radii of the cylinder and the thickness of the volume element $L=d z$ expressed as:

$$
V=S \cdot L=\pi\left(r_{o}^{2}-r_{i}^{2}\right) d z,
$$

where $r_{o}$ and $r_{i}$ are the external and internal radii of the cylinder, respectively. Heat lost by convection and radiation is expressed based on an overall heat transfer coefficient (OHTC) on the outer and inner surfaces of the specimen defined as $h_{o}$ and $h_{i}$, respectively. However, the environmental temperature of the outer surface $T_{o}$ is different from the inner surface $T_{i}$ due to the fact that the outer surface is completely exposed to the environment, $T_{o}$ remains constant, making 
it possible for heat transfer by natural radiation and convection, whereas the inner surface is considered to be a closed cavity in which air is confined and not completely still, limiting natural convection heat transfer by natural convection, and the governing mechanism therefore being radiation heat transfer, this causes the value of the $T_{i}$ to increase during heating, but always below the temperature of the specimen, generating a temperature gradient towards the interior. From this, we have:

$$
Q_{l}=h_{i}\left(T-T_{i}\right) A_{i}+h_{o}\left(T-T_{o}\right) A_{o},
$$

where $A_{i}=2 \pi r_{i} d z$ is the inner surface, and $A_{o}=2 \pi r_{o} d z$ is the outer surface of the specimen.

Finally, the heat generated by Joule heating [10] $Q_{g}$ is associated with the energy generated by the passage of electric current and is defined through Watt's law as:

$$
Q_{g}=I^{2} R_{e}
$$

where $I$ is the intensity of electric current, $R_{e}$ is the electric resistance that can be expressed in terms of resistivity $\rho_{e}$ or electrical conductivity $\sigma_{e}$, such as:

$$
R_{e}=\rho_{e} \frac{L}{S}=\frac{1}{\sigma_{e}} \frac{L}{S} .
$$

Consequently, the resistance of the volume element is:

$$
R_{e}=\left(\frac{1}{\sigma_{e}}\right) \frac{d z}{\pi\left(r_{o}^{2}-r_{i}^{2}\right)} .
$$

Substituting the Eqs. (2) to (7), in Eq. (1), we have the governing equation for the problem of Newtonian heating by the Joule effect:

$$
\begin{gathered}
\rho C_{p} \pi\left(r_{o}^{2}-r_{i}^{2}\right) d z \frac{d T}{d t}=\frac{I^{2}}{\sigma_{e}}\left[\frac{d z}{\pi\left(r_{o}^{2}-r_{i}^{2}\right)}\right]- \\
-h_{i}\left(T-T_{i}\right) 2 \pi r_{i} d z-h_{o}\left(T-T_{o}\right) 2 \pi r_{o} d z,
\end{gathered}
$$

dividing by $\rho C_{p} \pi\left(r_{o}^{2}-r_{i}^{2}\right) d z$,

$$
\begin{aligned}
\frac{d T}{d t} & =\left(\frac{I^{2}}{\sigma_{e}}\right)\left(\frac{1}{\rho C_{p}}\right)\left[\frac{1}{\pi\left(r_{o}^{2}-r_{i}^{2}\right)}\right]^{2}- \\
& -\frac{2\left[h_{i}\left(T-T_{i}\right) r_{i}+h_{o}\left(T-T_{o}\right) r_{o}\right]}{\rho C_{p}\left(r_{o}^{2}-r_{i}^{2}\right)} .
\end{aligned}
$$

Eq. (9) is a differential equation describing the heating rate, considering accumulated heat, heat generated by the Joule effect, heat dissipated by convection and radiation toward the heating medium.

\subsection{Thermal Dilatation}

Temperature changes cause thermal dilatation, expansion or contraction, in materials. In most of these, unitary thermal strain [11] $\varepsilon_{T}$ is expressed in terms of temperature change such as:

$$
\varepsilon_{T}=\alpha \cdot \Delta T,
$$

where $\alpha$ is the linear thermal expansion coefficient and $\Delta T$ is the temperature change regarding a reference temperature.

By analysing the thermal strain of the volume element of Fig. 1 and considering the real strain $\varepsilon$ [12] in terms of area we have:

$$
\varepsilon=\ln \left(\frac{A}{A^{\prime}}\right)=\ln \left(\frac{r_{o}^{2}-r_{i}^{2}}{r_{o}^{\prime 2}-r_{i}^{\prime 2}}\right) .
$$

Assuming that there are no temperature gradients in the radial direction, it is expected that the temperature in the volume element be uniform as will the linear thermal expansion, maintaining the difference between the out and inner radii $\Delta r$. Based on the above, Eqs. (10) and (11) are equalized at the strain value, obtaining an expression for the instant outer radius $r_{o}^{\prime}$ according to temperature change and the linear thermal expansion coefficient such that:

$$
r_{o}^{\prime}=\frac{2 r_{o}-\Delta r}{2 \cdot \exp (-\alpha \cdot \Delta T)}+\frac{\Delta r}{2} .
$$

Dilatation strain $\varepsilon_{d}$ is calculated based on the relationship of the instant diametric change $\Delta D^{\prime}$ and initial diameter $D_{o}$ through:

$$
\varepsilon_{d}=\frac{\Delta D^{\prime}}{D_{o}}=\frac{r_{o}^{\prime}-r_{o}}{r_{o}}
$$

\subsection{Method of Solution}

The proposed model in Eq. (9) may be solved analytically by considering constant thermophysical and electric properties; however, this does not happen in reality. Consequently, a solution of this type would be inaccurate. To provide a correct solution to the model it is necessary to consider the thermophysical and electrical properties of the material, and current intensity based on temperature and time: $\rho(T), C_{p}(T)$, $\sigma(T)$ and $I(t)$, respectively, making Eq. (9) take the form of a nonlinear differential equation whose analytic solution is not easily obtained. Consequently, it is proposed to use an approximate solution based on the explicit finite difference method (EFDM). In the 
EFDM, temperature profile is a function of the time and temperature and is calculated 'back time'. The method of solution was codified in the Scilab freelicense software ${ }^{\circledR}$ for which the differential forward form was used ahead of temperature time $d T / d t$ [13] as:

$$
\frac{d T}{d t}=\frac{T^{n+1}-T^{n}}{\Delta t},
$$

where $T^{n+1}$ is temperature future time $t^{n+1}$ and $T^{n}$ temperature in actual time $t^{n}$. Substituting Eq. (14) in Eq. (9) and employing the EFDM we have,

$$
\begin{aligned}
T^{n+1}= & {\left[\frac{I^{2}}{\sigma \rho C_{p}}\left(\frac{1}{\pi\left(r_{o}^{2}-r_{i}^{2}\right)}\right)^{2}-\frac{2}{\rho C_{p}\left(r_{o}^{2}-r_{i}^{2}\right)} .\right.} \\
& \left.\cdot\left[h_{o}\left(T^{n}-T_{o}\right) r_{o}+h_{i}\left(T^{n}-T_{i}\right) r_{i}\right]\right] \Delta t+T^{n} .
\end{aligned}
$$

Eq. (15) describes the evolution of the temperature field according to thermophysical and electrical properties, the heat transfer coefficient and the intensity of electric current, depending on the temperature, time and specimen dimensions.

\section{EXPERIMENTAL DEVELOPMENT}

For the development of the experimental tests a Joule heating device was built using instrumentation with a source of power of $2.25 \mathrm{~kW}$ coupled to a copper conductor jaw system to supply power. The jaws were cooled by means of a constant water flow in order to keep the ends of the specimen at a temperature of approximately $40{ }^{\circ} \mathrm{C}$; ensuring the concentration of the thermal field in the middle of the specimen. The temperature was measured by means of an infrared

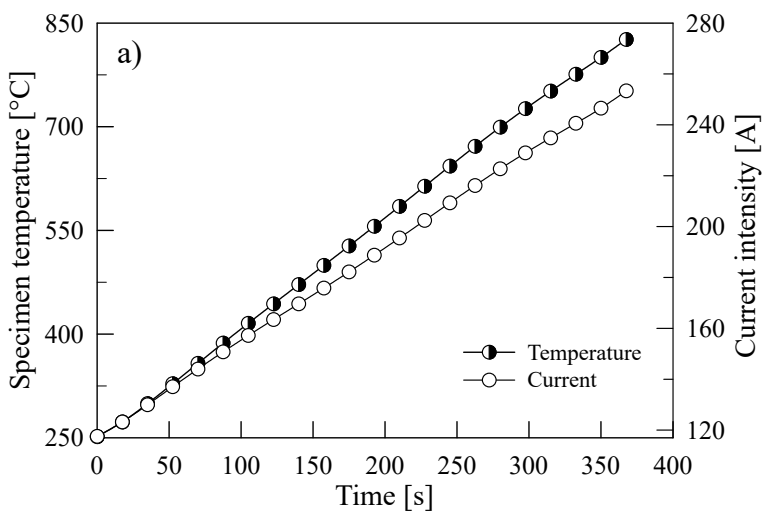

pyrometer based on a temperature of $250{ }^{\circ} \mathrm{C}$. In turn, the dilatation in the radial direction was measured using micrometer laser with a resolution of $\pm 1.0 \mu \mathrm{m}$. The devices used were controlled by a CompactRio NI cRIO 9075 data acquisition system. The experimental device can be seen in Fig. 2.

Specimens of an AISI 304 stainless steel were machined for the heating tests according to Fig. 1. The specimens were heated to a maximum temperature of $850{ }^{\circ} \mathrm{C}$ with different heating rates: $0.75^{\circ} \mathrm{C} \mathrm{s}^{-1}, 1.08$ ${ }^{\circ} \mathrm{C} \mathrm{s}^{-1}$, and $1.58{ }^{\circ} \mathrm{C} \mathrm{s}^{-1}$. Later, they were cooled to room temperature under natural convection conditions with still air and without passage of electric current. During heating, time, thermal response and variation of diameter were measured and acquired.

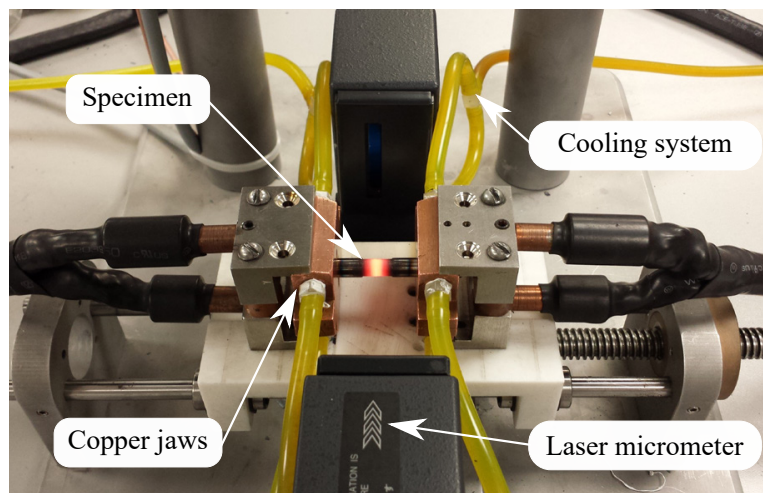

Fig. 2. Experimental Joule effect heating device

\section{RESULTS}

\subsection{Experimentation}

Results of the tests are shown in Fig. 3: a) thermal and current intensity history and b) strain percentage by dilatation $\% \Delta D^{\prime} / D_{o}$. From Fig. 3a we can see the

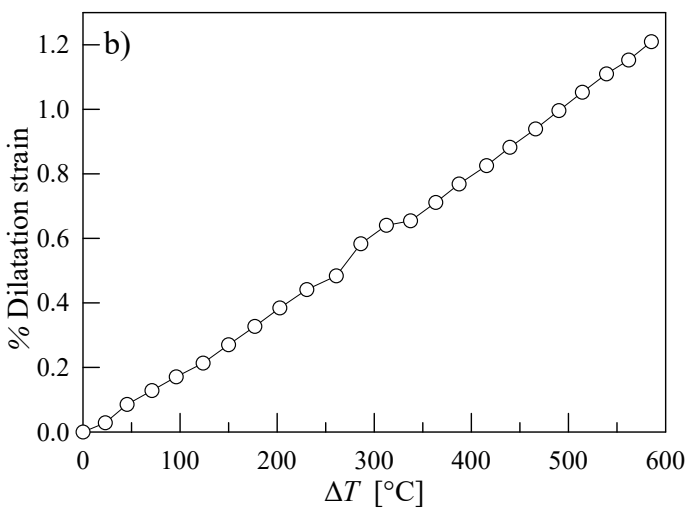

Fig. 3. a) Thermal history and current intensity supplied as a function of time and, b) percentage of strain from dilatation as a function of temperature change, in a hollow cylindrical specimen heated by the Joule effect to an average heating rate of $1.58{ }^{\circ} \mathrm{C} \mathrm{s}^{-1}$ 
thermal history measured in the middle section on the outer surface of the specimen, where it can be seen that temperature measurement starts when an approximate temperature of $250^{\circ} \mathrm{C}$ is reached and continues up to a temperature of $850^{\circ} \mathrm{C}$, in approximately 380 seconds, obtaining an average heating rate of $1.58{ }^{\circ} \mathrm{C} \mathrm{s}^{-1}$. In the same Fig. $3 \mathrm{a}$ the current intensity supplied to the specimen in shown with regards to time. The current intensity required to reach an initial temperature of $250{ }^{\circ} \mathrm{C}$ is less than $120 \mathrm{~A}$, which increases in direct proportion to the temperature until it reaches an approximate value of $260 \mathrm{~A}$. The behaviour of the current intensity is similar to that of the thermal history, there being a linear relationship between the current supplied and the specimen temperature.

In contrast, the percentage of dilatation strain is shown in Fig. $3 \mathrm{~b}$ as a function of the temperature change with regard to initial temperature $\Delta T=T-250$. As with temperature and current intensity, the percentage of dilatation strain presents a behaviour directly proportional to the $\Delta T$ value until reaching a change when approaching $600^{\circ} \mathrm{C}$.

\subsection{Thermal Boundary Condition (TBC)}

As indicated in the model, Thermal Boundary Conditions between the outer and inner surfaces with the environment were defined based on an overall heat transfer coefficient, $h_{o}(T)$ and $h_{i}(T)$. In the case of the outer surface, the value of the coefficient must be determined based on the temperature, as the heat flow of the specimen toward the environment is given by the contribution of heat lost by convection and radiation:

$$
h_{o}=h_{c}+h_{r},
$$

where $h_{c}$ is the heat transfer coefficient by convection and $h_{r}$ is the radiation coefficient. The heat transfer coefficient from natural convection is obtained based on the definition of the Nusselt dimensionless number, $N u_{D}$ such as:

$$
h_{c}=\frac{N u_{D} \cdot k_{\text {air }}}{D_{o}},
$$

where $k_{\text {air }}$ is the thermal conductivity of the air and $D_{o}$ is the initial outside diameter of the specimen. The value of $N u_{D}$ is obtained based on the empirical correlation developed by Rebrov [14], for horizontal cylinders with diameters between 1.31 and $9.9 \mathrm{~mm}$ subjected to natural convection,

$$
N u_{D}=\left[0.98-0.01\left(\log G r_{D} \operatorname{Pr}\right)^{2}\right]\left(G r_{D} \operatorname{Pr}\right)^{x},
$$

where $G r_{D}$ is the Grashof dimensionless number evaluated with the outer diameter of the specimen and $\operatorname{Pr}$ is the Prandtl number while the exponent $x$ is expressed as:

$$
x=0.14+0.015 \log \left(G r_{D} \operatorname{Pr}\right) .
$$

In contrast, the radiation transfer coefficient $h_{r}$ is defined as [15]:

$$
h_{r}=\varepsilon \cdot \sigma\left[\frac{\left(T^{4}-T_{o}^{4}\right)}{T-T_{o}}\right],
$$

where $\varepsilon$ is the material emissivity and $\sigma$ is the Stefan Boltzmann constant and temperatures are expressed in Kelvin.

Fig. 4 shows the heat transfer coefficients as a function of the temperature for the outer surface of the specimen. The natural convection coefficient is practically constant over the whole temperature range, while the radiation coefficient increases exponentially with the rising specimen temperature, indicating that heat transfer by radiation is the high-temperature governing mechanism.

To obtain the TBC inside the specimen, it was necessary to determine the environmental temperature inside the specimen. For this, the temperature of the middle was measured with a thermocouple placed inside the specimen as shown in Fig. 1.

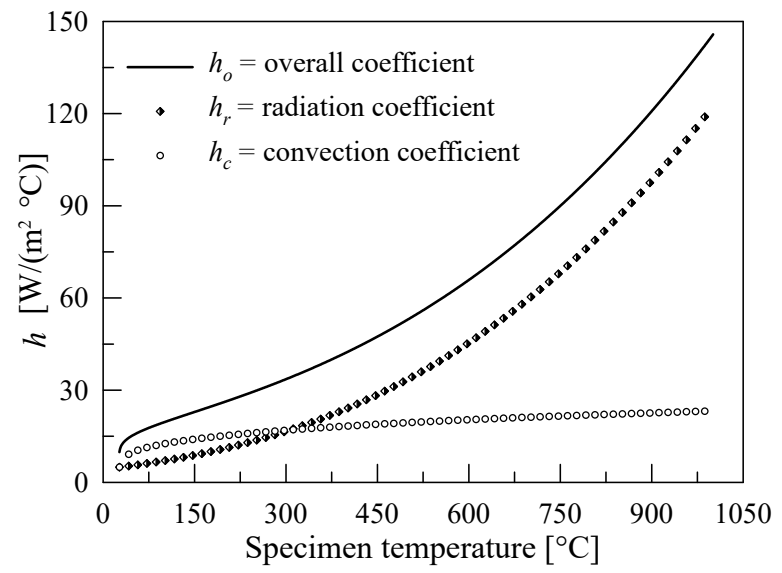

Fig. 4. Comparison between heat transfer coefficients by convection $h_{c}$, radiation $h_{r}$, and overall coefficient ho to estimate boundary condition convective in the outer surface of the specimen

However, the temperature acquired by the thermocouple not only considers heat transfer by convection between the thermocouple and the environment but also radiation between the inner surface and the tip of the thermocouple due to the difference of temperature between the surface and the 
environment. This causes the temperature acquired by the thermocouple to be an average temperature between the inner surface and the environment. For this the inner temperature is calculated using a balance of energy on the surface of the thermocouple, equalling the heat gained through radiation with heat lost by convection [16]:

$$
\dot{q}_{c o n v, \rightarrow t p}=\dot{q}_{r a d, \leftarrow t p},
$$

where the arrow shows the direction of heat flow, $\rightarrow t p$ toward the thermocouple, $\leftarrow t p$ from the thermocouple,

$$
h\left(T_{i}-T_{t p}\right)=\varepsilon_{t p} \sigma\left(T_{t p}^{4}-T^{4}\right),
$$

where $\varepsilon_{t p}$ is the emissivity of the thermocouple. For the inner temperature $T_{i}$,

$$
T_{i}=T_{t p}+\frac{\varepsilon_{t p} \sigma\left(T_{t p}^{4}-T^{4}\right)}{h},
$$

where, $T_{t p}$ is the temperature acquired by the thermocouple of the centre, whose location is shown in Fig. 1, $T$ is the specimen temperature and $h$ is the heat transfer coefficient by convection between the tip of the thermocouple and the inner surface of the specimen. Fig. 5 shows a) the comparison between specimen temperature $T$, thermocouple temperature $T_{t p}$ and the average inner temperature $T_{i}$ calculated based on Eq. (23) as a function of heating time, and b) temperature of the inner room as a function of the specimen temperature.

Finally, to estimate TBC inside $h_{i}$ the inverse heat conduction problem (IHCP) was solved [17] and [18]. Eq. (9) was resolved for $h_{i}$, supplying the thermal history of the specimen, the temperature of the inner and outer room, the current intensity history, electrical and thermophysical properties dependent on the temperature for AISI 304 stainless steel, this data was obtained from the properties reported in the literature [19] and [20]. The IHCP was solved by means of a nonlinear Beck method [21] and [22]; the result is shown in Fig. 6.

In Fig. 6 you can see the behaviour of the overall heat transfer coefficient between the inner surface of the specimen and the environment. When the heating starts, the inner environment has a growing heat extraction capacity up to a temperature of $550{ }^{\circ} \mathrm{C}$, mainly due to a temperature difference between the environment and the specimen. From this point, extraction remains practically constant around $315 \mathrm{~W} /\left(\mathrm{m}^{2}{ }^{\circ} \mathrm{C}\right)$ until reaching a temperature approaching $800{ }^{\circ} \mathrm{C}$, before reaching the maximum specimen temperature. At this point, the extraction capacity drops slightly due to heat saturation of the inner environment and for the heat loss due to convection-radiation in the opposite side of the specimen. This is a particular IHCP solution for the internal heat transfer coefficient due to the dependence of the inner geometry of the specimen.
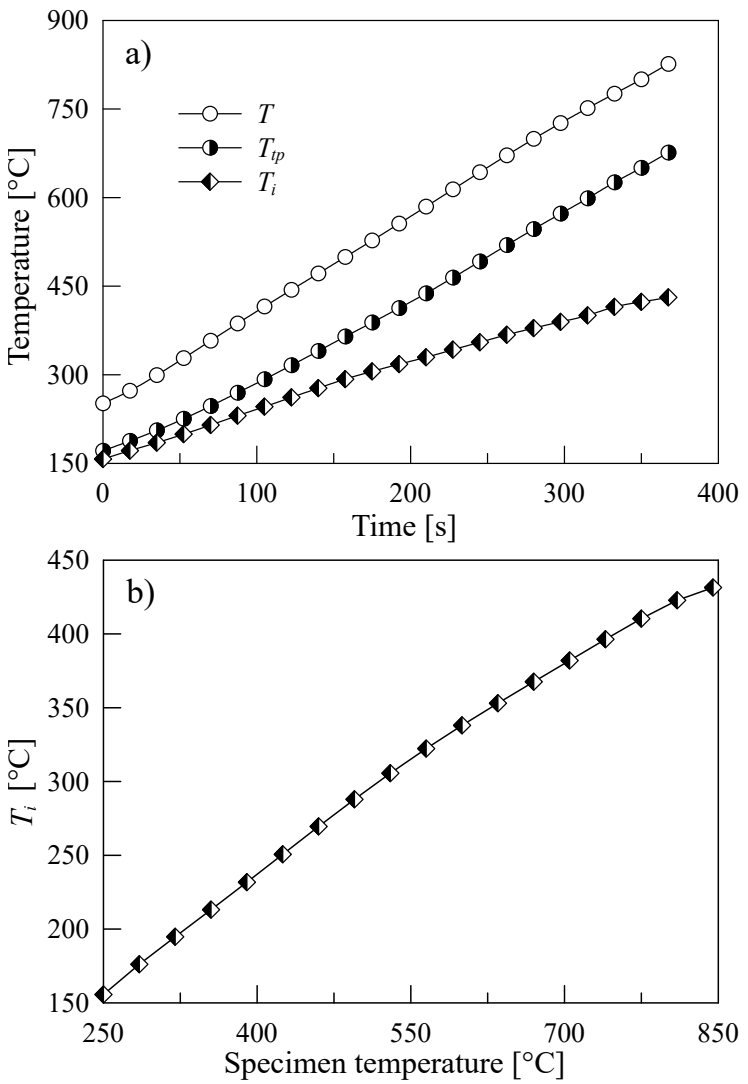

Fig. 5. a) Comparison between specimen temperature $T$, thermocouple temperature $T_{t p}$ and the average inner temperature $T_{i}$, as calculated based on Eq. (23), and b) temperature of the inner room as a function of the temperature of the specimen

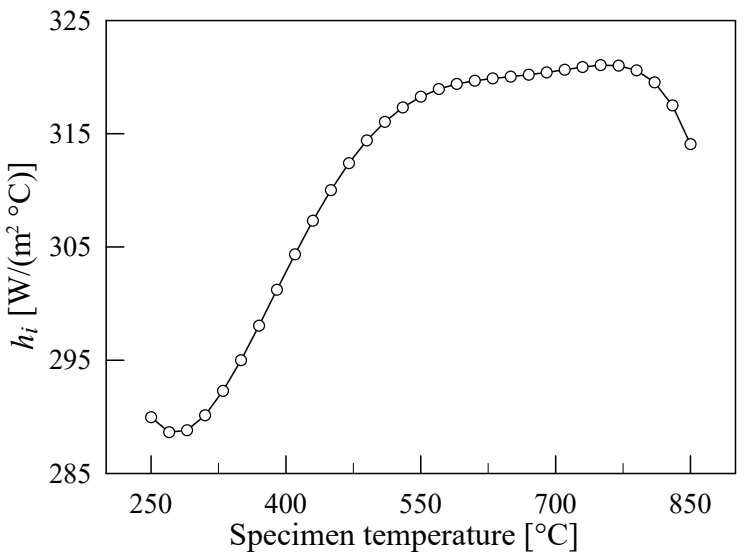

Fig. 6. Heat Transfer coefficient $h_{i}$ for the inner surface, as a function of specimen temperature calculated based on IHCP solution 


\subsection{Determining the Linear Thermal Expansion Coefficient}

The linear thermal expansion coefficient is determined based on dilatation strain and experimentally measured diameter change, considering that the thermal strain $\varepsilon_{T}$ is equal to dilatation strain $\varepsilon_{d}$ of the specimen.

$$
\alpha=\frac{1}{D_{o}} \frac{\Delta D_{o}^{\prime}}{\Delta T}
$$

where $\Delta T$ is the change of temperature, expressed as:

$$
\Delta T=T^{n+1}-T_{r},
$$

where $T^{n+1}$ is instantaneous temperature in future time $t^{n+1}$, for each instantaneous outside radius value $r_{o}^{\prime}$, while $T_{r}$ is the reference temperature corresponding to the initial outside radius $r_{o} ; T_{r}$ is a constant that coincides with initial specimen temperature. The linear thermal expansion coefficient obtained from the Eq. (24) and experimental measurements is shown in Fig. 7.

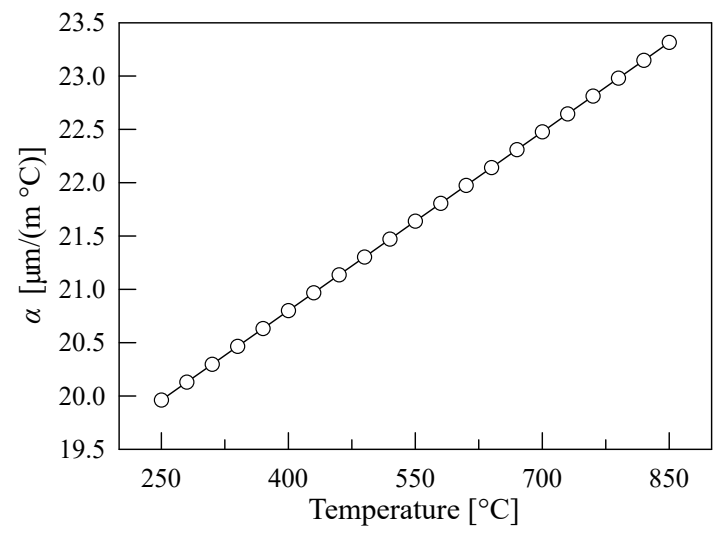

Fig. 7. Experimental linear thermal expansion coefficient $\alpha$, as a function of temperature for an AISI 304 stainless steel

Fig. 7 shows that the linear thermal expansion coefficient maintains linear behaviour regarding temperature and because it was calculated based on the temperature change $\Delta T$ we have a different value for each of the future temperatures $T^{n+1}$. The average thermal expansion coefficient $\bar{\alpha}$ is $21.64 \mu \mathrm{m} /\left(\mathrm{m}^{\circ} \mathrm{C}\right)$, corresponding to stainless steel with an austenitic structure [23].

\section{VALIDATION OF THE MATHEMATICAL MODEL}

Fig. 8 shows the comparison between the experimental thermal history and that obtained by mathematical model for a heating rate of: $0.75^{\circ} \mathrm{Cs}^{-1}, 1.08^{\circ} \mathrm{C} \mathrm{s}^{-1}$, and $1.58{ }^{\circ} \mathrm{C} \mathrm{s}^{-1}$. As mentioned previously, the model is supplied with thermophysical and electrical properties dependent on the temperature and heat transfer coefficients $h_{o}$ and $h_{i}$ obtained for Rebrov's empirical correlation and solving the inverse IHCP problem, respectively. Fig. 8 shows the thermal history obtained with the mathematical model, which acceptably concurs with the thermal history obtained experimentally. This validates the TBC established for each of the surfaces of the specimen, confirming the assumption in terms of radiative contribution to the interior is significant and accurate, making it possible to appropriately predict the evolution of the thermal field during heating by the Joule effect.

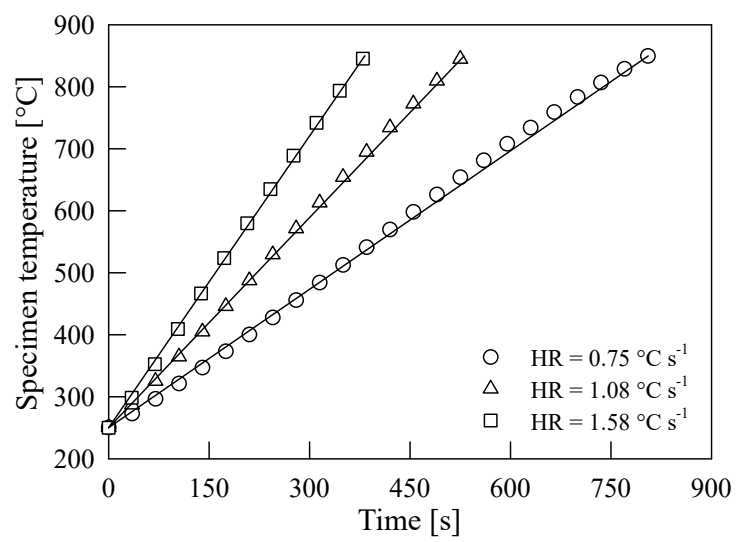

Fig. 8. Shows the experimental thermal history (symbols) and simulated thermal history (lines) for the heating rates of:

$0.75^{\circ} \mathrm{C} \mathrm{s}^{-1}, 1.08{ }^{\circ} \mathrm{C} \mathrm{s}^{-1}$, and $1.58{ }^{\circ} \mathrm{C} \mathrm{s}^{-1}$ for an AISI 304 stainless steel

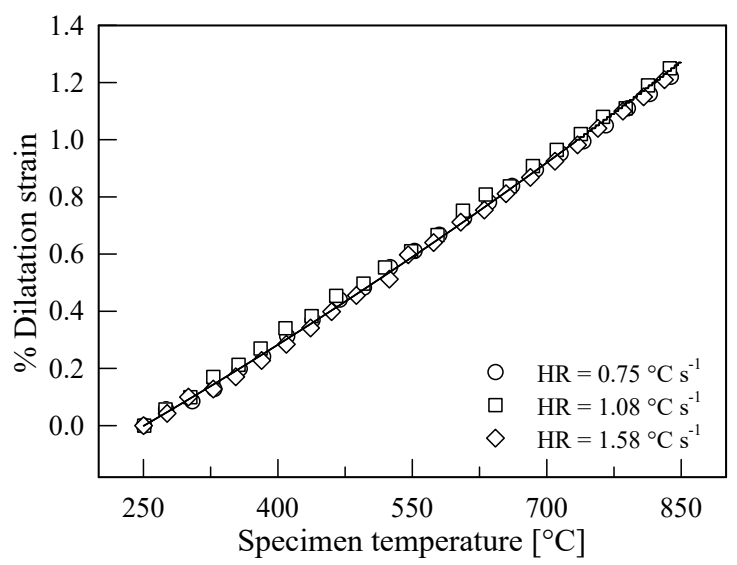

Fig. 9. Dilation strain experimental (dots) and simulated values with the model (line) for the heating rates of: $0.75{ }^{\circ} \mathrm{C} \mathrm{s}^{-1}$, $1.08{ }^{\circ} \mathrm{C} \mathrm{s}^{-1}$, and $1.58{ }^{\circ} \mathrm{C} \mathrm{s}^{-1}$ for AISI 304 stainless steel

Steel dilatation measurements were used to validate the mathematical model, and Fig. 9 shows the dilation strain for different heating rates for experimental (dots) and simulated values with the model (line). The dilatation strain is referenced to the outer diameter value to a temperature of $250{ }^{\circ} \mathrm{C}$ for 
each heating rate. As with the thermal history, a linear behaviour is observed where the adjustment between experimental values and those simulated with the model is acceptable. From the same figure it can be inferred that the linear thermal expansion coefficient is independent of the heating rate.

\section{SENSITIVITY ANALYSIS}

A sensitivity analysis of the mathematical model was performed to determine the effect of the critical input variable on the specimen's thermal history. For this the function of electrical conductivity $\sigma_{e}(T)$ was employed, modifying the function $\pm 20 \%$ and $\pm 40 \%$ with respect to the original value, this response was called the "medium scenario", trying to emulate a material with greater and weaker conductivity to determine its influence on the thermal history of the specimen. Likewise, the thickness of the dilatometric specimen used was referred to as the difference between the outside and the inside radii $\Delta r$. This parameter was selected due to the mass factor and its influence on thermal history, for which the outer diameter was modified from $2.9 \mathrm{~mm}$ and $4.1 \mathrm{~mm}$, thereby obtaining different wall thicknesses for the dilatometric specimen. The other input variables such as thermophysical properties and electric current history were set in the sensitivity analysis. Fig. 10 shows the family of curves for electrical conductivity $\sigma_{e}(T)$ and the current history used in the sensitivity analysis.

\subsection{Input Variables}

Electrical conductivity is a major input variable in the model because, under the physical principal of Joule

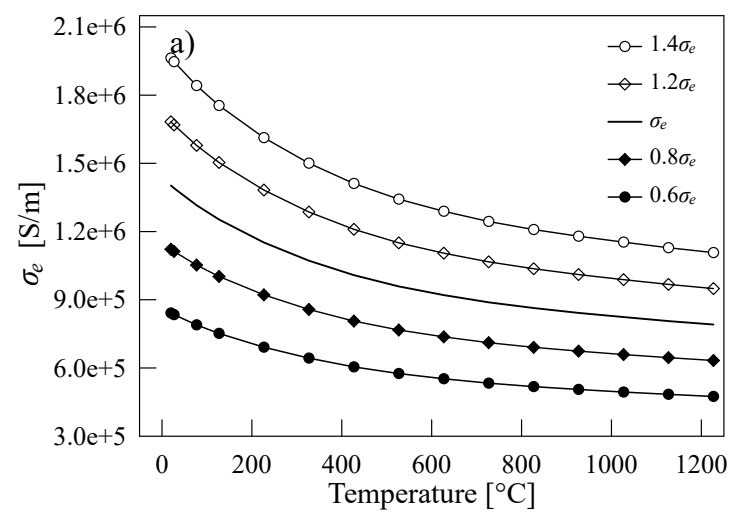

heating, the dilatometric specimen acts as electrical resistance to the circuit, so that a small change in its value is reflected as an increase or decrease of the required electric current intensity. Likewise, the thickness of the specimen delimits the heating conditions due to the amount of mass to be heated and the contact area through which the electric current circulates. Both variables were modified and analysed with regard to simulated thermal history, comparing the results with the medium scenario response. Fig. 11 shows the effects of electrical conductivity and the thickness of the specimen on simulated thermal history.

Fig. 11a shows that when using conductivity values below $\sigma_{e}$, the thermal response suddenly accelerates, reaching elevated temperatures in short times. In this regard, the heating rate is greater than the medium scenario rate. Later, the heating rate changes, approaching the medium scenario rate of $0.66{ }^{\circ} \mathrm{C} \mathrm{s}^{-1}$ until a temperature of $900{ }^{\circ} \mathrm{C}$ is reached. As indicated, when reducing electrical conductivity, the resistivity of the material increases. The abrupt change observed at the beginning of the curves, with conductivity below $\sigma_{e}$ owes to current history $I(t)$, Fig. $10 \mathrm{~b}$, which is the electric current history used in the medium scenario and in whole analysis scenarios. A material with greater resistivity requires less current to reach a specific temperature. However, by keeping the same current history, the material tends to heat at greater rates, as seen in this case; the model tries to maintain the restriction in regards to heating rate, stabilizing the thermal response with regards to the medium scenario response. In the opposite case, for conductivity greater than $\sigma_{e}$, the temperature drops below the starting temperature $\left(350{ }^{\circ} \mathrm{C}\right)$, this light undercooling is more apparent when the

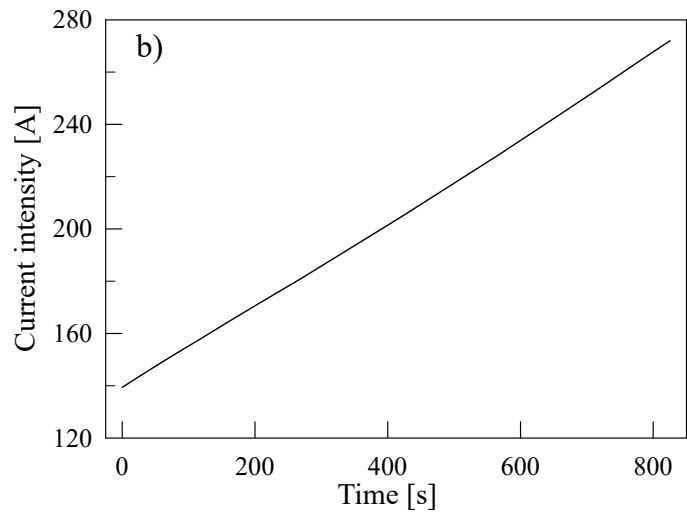

Fig. 10. a) Electrical conductivity for an AISI 304 stainless steel as function of the temperature; the figure shows the family of curves used in sensitivity analysis, the value of the original electrical conductivity varying $\pm 20 \%$ and $\pm 40 \%$, the solid line indicated the electrical conductivity used for the medium scenario, and b) electric current history used for sensitivity analysis 
electrical conductivity is higher, due to electrical resistivity reducing without opposing the passage of current, which is insufficient to maintain and raise the temperatures above $350{ }^{\circ} \mathrm{C}$. Furthermore, the boundary conditions are fundamental to heating of the specimen due to the fact that the amount of heat lost by the boundaries is greater than the amount generated by the Joule heating, reducing the temperature of the specimen until the current intensity rises until reaching and raising the initial temperature. However, for the same current history, conductivity values above $\sigma_{e}$ do not allow the temperature seen in the medium scenario to be reached, because when increasing conductivity, resistivity drops, making the system require a greater amount of current to reach the established temperature of $900{ }^{\circ} \mathrm{C}$. In this case, when conductivity is equal to $1.4 \sigma_{e}$ the temperature to be reached at the same time or with the same current intensity is barely $734^{\circ} \mathrm{C}$.

In contrast, wall thickness influences the thermal response of the specimen, as shown in Fig. 11b. In this figure, thicknesses between $0.4 \mathrm{~mm}$ and 1.6 $\mathrm{mm}$ are listed, considering that the thickness of the specimen for the medium scenario is $1 \mathrm{~mm}$. When specimen thickness is below $1 \mathrm{~mm}$, thermal response is incredibly fast, especially when the $\Delta r$ is equal to $0.4 \mathrm{~mm}$, the temperature then rises rapidly to $800^{\circ} \mathrm{C}$ in just 18 seconds, averaging a heating rate of $25{ }^{\circ} \mathrm{C} \mathrm{s}^{-1}$, well above the rate of the medium scenario rate of $0.66{ }^{\circ} \mathrm{Cs}^{-1}$. Later, the rate continuously drops to approximately $0.66{ }^{\circ} \mathrm{Cs}^{-1}$ until reaching the temperature of $900^{\circ} \mathrm{C}$, the same effect can be seen for a thickness of $0.8 \mathrm{~mm}$, but to a lesser degree. These changes are directly associated with the contact area. Upon reducing the area, current density increases, making the current concentrate and increasing

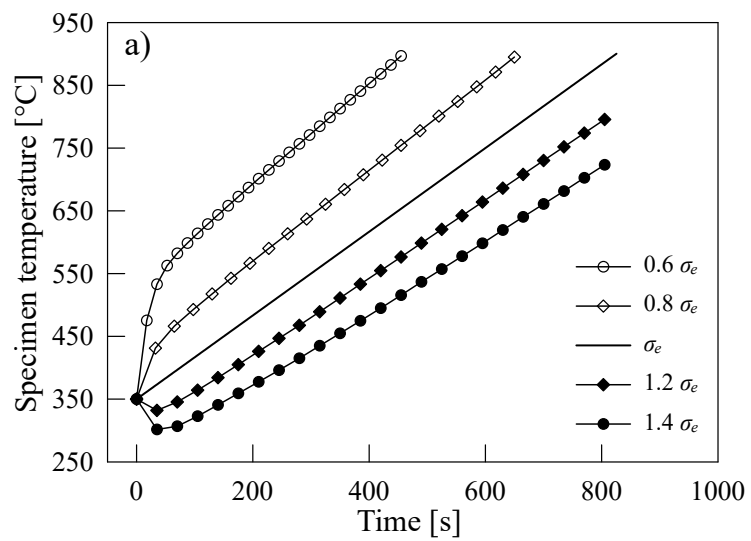

the temperature of the specimen considerably. In contrast, when thickness increases above $1 \mathrm{~mm}$, the current supply is insufficient to maintain the initial temperature of $350{ }^{\circ} \mathrm{C}$, and consequently to increase it. In this case, the heat extracted by the surfaces of the specimen is greater than the heat generated and the temperature drops to $250^{\circ} \mathrm{C}$ for a thickness of $1.6 \mathrm{~mm}$. After a time, the current continues to increase, Fig. $10 \mathrm{~b}$, increasing the temperature of the specimen to above $350^{\circ} \mathrm{C}$. However, with the same current history, the specimen does not manage to heat up to the temperature of $900{ }^{\circ} \mathrm{C}$ like in the medium scenario. When the thickness of the specimen is greater, the density of the current is reduced, preventing the material from heating up the same way.

As has been noted, electrical conductivity and specimen thickness have a strong impact on thermal response, both variables are critical to meeting the thermal heating conditions due to the Joule effect. Therefore, both material selection and specimen dimensions are essential to adequately design a thermal cycle.

\section{PREDICTIONS}

Finally, the mathematical model was employed to carry out a series of predictions on a metallic alloy other than AISI 304 stainless steel, a super nickel-based Hastelloy ${ }^{\circledR}$ C-276, which is characterized for having high mechanical resistance at high temperatures. Fig. 12 shows the thermal behaviour of Hastelloy ${ }^{\circledR}$ C-276 heated by the Joule effect to a temperature of $900{ }^{\circ} \mathrm{C}$, in Fig. 12a shows thermal response, 12b the electric current history, and $12 \mathrm{c}$ the percentage of dilatation strain. To predict the thermal behaviour of Hastelloy ${ }^{\circledR}$ C-276 the same heating rate used on the stainless steel

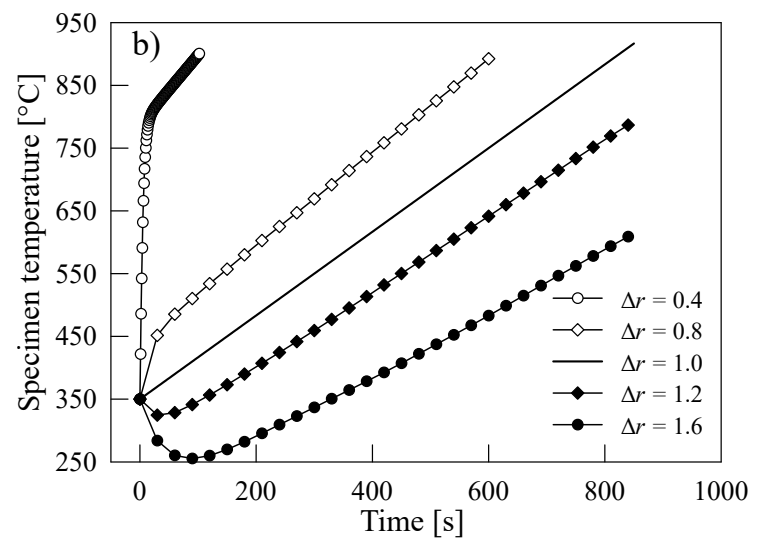

Fig. 11. a) Simulated thermal response from the specimen using different values of electrical conductivity for an AISI 304 stainless steel, and b) simulated thermal response from the specimen using different values of thickness of the specimen for an AISI 304 stainless steel; the solid line shows the thermal response of medium scenario, the heating rate was $0.66{ }^{\circ} \mathrm{Cs}^{-1}$ 

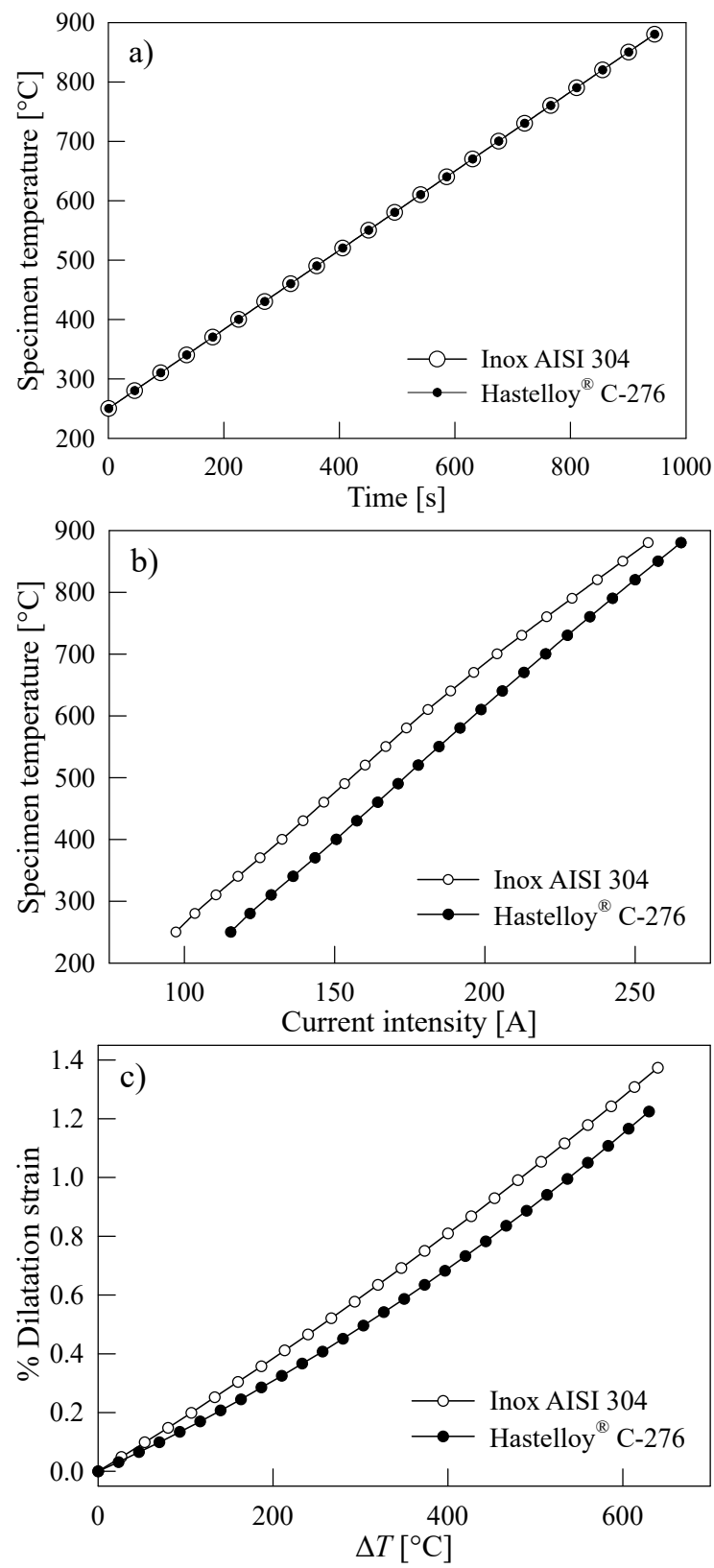

Fig. 12. a) Prediction and comparison of thermal history, b) current history, and c) percentage of dilatation strain of an alloy base nickel Hastelloy ${ }^{\circledR}$ C-276 with regards to AISI 304 stainless steel

$\left(0.66^{\circ} \mathrm{C} \mathrm{s}^{-1}\right)$ was applied, along with similar specimen dimensions with a thickness of $\Delta r 1 \mathrm{~mm}$.

Fig. 12a shows the comparison between the thermal history of the Hastelloy and stainless steel, showing that there is no difference in thermal response for both materials, regardless of the thermophysical and electrical properties. This is due to the heating rate remaining fixed at $0.66{ }^{\circ} \mathrm{C} \mathrm{s}^{-1}$ in both cases. The model sample has the capacity to adjust the heating rate and simultaneously calculate the electric current required to heat the specimens at a final temperature of $900^{\circ} \mathrm{C}$. Fig. $12 \mathrm{~b}$ shows the comparison between the current histories predicted by the model, both materials initial temperatures are $250^{\circ} \mathrm{C}$. Unlike the thermal response, it can be observed that to heat a Hastelloy specimen to $900{ }^{\circ} \mathrm{C}$ requires a greater current. The behaviour is similar and mostly linear for the two materials. Finally, Fig. 12c shows the behaviour of the dilatation strain of both materials. It can be noted that the behaviour is not completely linear, which generates a curvature as the temperature rises. Although, specimens with the same thickness are considered, the response is different due to the thermal expansion coefficient. Stainless steel has greater strain by dilatation when reaching $900{ }^{\circ} \mathrm{C}$ than the Hastelloy does. These predictions are useful for estimating, establishing, and designing heating conditions for a conductor material and specifying the amount of electric current and thickness required to reach the temperature given in order to study the mechanical-electrical-thermal behaviour of the material.

\section{CONCLUSIONS}

Based on the results obtained it has been shown that it is possible to develop a mathematical Joule heating model for a dilatometric specimen, by estimating the thermal history, electric current history, and percentage of dilatation strain for a hollow cylindrical specimen with thermophysical and electrical properties, depending on the temperature. It was also shown that it is possible to estimate the thermal boundary conditions using an empirical correlation and the solution to the IHCP to determine the overall heat transfer coefficient in the inner and outer surfaces of the specimen, taking into account the phenomena of convection and radiation. Likewise, the model was satisfactorily validated by comparing it with the thermal history and percentage of dilatation strain by experimental with simulated responses with the mathematical model, to data acquired at a different heating rate. Additionally, a sensitivity analysis was conducted to determine the effect of the critical variables of the model, such as electrical conductivity and the thickness of the specimen. The effect of electrical conductivity was observed on the thermal history, leading to the conclusion that the increase in conductivity hinders heating because the electrical resistance of the system decreases. In contrast, the thickness of the specimen has a strong influence on the heating rate, because when the thickness is decreased, the current density increases to rapidly 
achieve rates higher than those established. Finally, the mechanical-electrical-thermal behaviour of the nickelbased Hastelloy ${ }^{\circledR}$ C-276 was estimated, with electric properties similar to stainless steel and it was shown in the results that they were as expected, that is, a lesser temperature in the middle section and dilatation of the specimen for the same value of electric current supplied to the AISI 304 stainless steel specimen, due to the fact that Hastelloy has lower conductivity and linear thermal expansion than that of stainless steel.

\section{ACKNOWLEDGEMENTS}

M. Herrejón-Escutia would like to thank the National Council of Science and Technology of Mexico (CONACYT) for the scholarship (No. 267206) received for his doctoral studies. The authors are grateful to SEP-CONACYT and PRODEP for the support received through grants CB-256843 and UNISTMO-PTC-108, respectively.

\section{REFERENCES}

[1] García de Andrés, C., Caballero, F.G.., Capdevila, C., Álvarez, L.F. (2002). Application of dilatometric analysis to the study of solid-solid phase transformations in steels. Materials Characterization, vol. 48, no. 1, p. 101-111, D0l:10.1016/ S1044-5803(02)00259-0.

[2] Moravčík, R., Štefániková, M., Čička, R., Čaplovič, Ĺ., Kocúrová, K., Šturm, R. (2012). Phase transformations in high alloy cold work tool steel. Strojniški vestnik - Journal of Mechanical Engineering, vol. 58, no. 12, p. 709-715, Dol:10.5545/svjme.2012.531.

[3] Oliveira, F.L.G., Andrade, M.S., Cota, A.B. (2007). Kinetics of austenite formation during continuous heating in a low carbon steel. Materials Characterization, vol. 58, no. 3, p. 256-261, D0I:10.1016/j.matchar.2006.04.027.

[4] López-Martínez, E., Vázquez-Gómez, 0., Vergara-Hernández, H.J., Campillo, B. (2015). Effect of initial microstructure on austenite formation kinetics in high-strength experimental microalloyed steels. International Journal of Minerals, Metallurgy, and Materials, vol. 22, no. 12, p. 1304-1312, D0l:10.1007/s12613-015-1198-4.

[5] Vázquez-Gómez, 0., Barrera-Godínez, J.A., HernándezMorales, B., Vergara-Hernández, H.J., López-Martínez, E. (2012). Mathematical model of thermal and microstructural evolution during austempering of ductile iron. Materials Performance and Characterization, vol. 1, no. 1, p. 1-14, D0I:10.1520/MPC104384.

[6] López-Martínez, E., Hernández-Morales, J.B., Solorio-Díaz, G., Vergara-Hernández, H.J., Vázquez-Gómez, O., GarnicaGonzález, P. (2013). Prediction of hardness profiles in medium and low carbon steel jominy probes. Mexican Journal of Chemical Engineering, vol. 12, no. 3, p. 606-616. (in Spanish)

[7] Mohanty, R.R., Girina, O.A., Fonstein, N.M. (2011). Effect of heating rate on the austenite formation in low-carbon high-strength steels annealed in the intercritical region. Metallurgical and Materials Transactions A, vol. 42, p. 36803690, D0I:10.1007/s11661-011-0753-5.

[8] Vázquez-Gómez, 0., Barrera-Godínez J.A., Vergara-Hernández H.J. (2015). Kinetic study of austenite formation during continuous heating of unalloyed ductile iron. International Journal of Minerals, Metallurgy, and Materials, vol. 22, no. 1, p. 27-31, DOI:10.1007/s12613-015-1039-5.

[9] Herrejón-Escutia, M., Arreola-Villa, S.A., Solorio-Díaz, G., Vázquez-Gómez, 0. (2016). Experimental determination of the electrical resistivity of a nickel base alloy during continuous heating by Joule effect, Investigative Compendium of Academia Journals Celaya, p. 2856-2860. (in Spanish)

[10] Holman, J.P. (1986). Heat Transfer. McGraw-Hill, Singapore.

[11] Gere, J.M., Goodno, B.J. (2013). Mechanics of Materials. Cengage Learning, Toronto.

[12] Dieter, G.E. (1986). Mechanical Metallurgy. McGraw-Hill, New York.

[13] Malek, A. (2011). Applications of nonstandard finite difference methods to nonlinear heat transfer problems, Belmiloudi, A. (ed.), Heat Transfer-Mathematical Modelling. Numerical Methods and Information Technology. InTech, p. 185-208, DOI:10.5772/14439.

[14] Boetcher, S.K.S. (2014). Natural Convection from Circular Cylinders. Springer, Cham, D0I:10.1007/978-3-319-08132-8.

[15] Poirier, G.H., Geiger, D.R. (2009). Transport Phenomena in Materials Processing. TSM Minerals Metals Materials, Pennsylvania.

[16] Cengel, Y.A. (2003). Heat Transfer a Practical Approach. McGraw-Hill, New York.

[17] Narazaki, M., Kogawara, M., Ming, Q., Watanabe, Y. (2009). Measurement and database construction of heat transfer coefficients of gas quenching. Strojniški vestnik - Journal of Mechanical Engineering, vol. 55, no. 3, p. 167-173.

[18] Özisik, M.N., Orlande H.R.B. (2000). Inverse Heat Transfer: Fundamentals and Applications. Taylor \& Francis, New York.

[19] Daw, J.E., Rempe, J.L., Knudson, D.L. (2009). Thermal properties of structural materials found in light water reactor vessels. Idaho National Laboratory (INL), Indaho, DOI:10.2172/974795.

[20] Ho, C.Y., Chu, T.K. (1977). Electrical resistivity and thermal conductivity of nine selected AISI stainless steels. Center for Information and Numerical Data Analysis and Synthesis, Indiana.

[21] Beck, J.V. (1970). Nonlinear estimation applied to the nonlinear inverse heat conduction problem. International Journal of Heat and Mass Transfer, vol. 13, no. 4, p. 703-716, D0I:10.1016/0017-9310(70)90044-X.

[22] Reinhardt, H.-J., Hào, D.N. (1994). Sequential approximation to nonlinear inverse heat conduction problems. Mathematical and Computer Modelling, vol. 20, no. 10-11, p. 189-200, DOI:10.1016/0895-7177(94)90177-5.

[23] Hawbolt, E.B., Chau, B., Brimacombe, J.K. (1983). Kinetics of austenite-pearlite transformation in eutectoid carbon steel. Metallurgical Transactions A, vol. 14, no. 9, p. 1803-1815, DOI:10.1007/BF02645550. 